\title{
Sample size calculations: should the emperor's clothes be off the peg or made to measure?
}

In this Research Methods and Reporting article, a type II error was incorrectly defined in the first paragraph of the section "What $\alpha$ and $\beta$ levels do you want?" (BMJ 2012;345:e5278, doi:10.1136/bmj.e5278). A type II error should have been defined as: "accepting [not "rejecting" as was published] the null hypothesis when the alternate hypothesis is true."

Cite this as: BMJ 2014;349:g5341

๑ BMJ Publishing Group Ltd 2014 